\title{
Circuit QED: Generation of two-transmon-qutrit entangled states via resonant interaction
}

\author{
Xi-Mei Ye ${ }^{1}$, Zhen-Fei Zheng ${ }^{3}$, Dao-Ming $\mathrm{Lu}^{1}$, and Chui-Ping Yang 2 果 \\ ${ }^{1}$ College of Mechanic and Electronic Engineering, \\ Wuyi University, Wuyishan, Fujiang 354300, China \\ ${ }^{2}$ KDepartment of Physics, Hangzhou Normal University, Hangzhou 310036, China and \\ ${ }^{3}$ Department of Physics, University of Science and Technology of China, Heifei 230026, China
}

(Dated: June 13, 2018)

\begin{abstract}
We present a way to create entangled states of two superconducting transmon qutrits based on circuit QED. Here, a qutrit refers to a three-level quantum system. Since only resonant interaction is employed, the entanglement creation can be completed within a short time. The degree of entanglement for the prepared entangled state can be controlled by varying the weight factors of the initial state of one qutrit, which allows the prepared entangled state to change from a partially entangled state to a maximally entangled state. Because a single cavity is used, only resonant interaction is employed, and none of identical qutrit-cavity coupling constant, measurement, and auxiliary qutrit is needed, this proposal is easy to implement in experiments. The proposal is quite general and can be applied to prepare a two-qutrit partially or maximally entangled state with two natural or artificial atoms of a ladder-type level structure, coupled to an optical or microwave cavity.

PACS numbers: 03.67.Bg, 42.50.Dv, 85.25.Cp
\end{abstract}

\section{INTRODUCTION AND MOTIVATION}

Entanglement is one of the most striking aspects of quantum mechanics. A system is entangled if its quantum state cannot be described as a direct product of states of its subsystems. Entanglement has many applications in quantum commuication and quantum information processing (QIP) [1-5]. In the last decade, quantum entanglement engineering has been widely studied with various physical systems. For instance, entanglement creation has been theoretically proposed with superconducting (SC) qubits coupled via Josephson junctions [6] or capacitors [7-9]. The focus of this work is on entanglement creation with a circuit-QED system, which has been considered as one of the leading candidates for QIP [10-17]. For the past years, based on circuit QED, much process has been made on entanglement generation with SC qubits. For instance, using SC qubits coupled to a single cavity or multiple resonators (hereafter, the terms cavity and resonator are used interchangeably), a number of theoretical proposals have been presented for realizing entangled states of SC qubits [10-12,18-23]. Moreover, various two-qubit or three-qubit entangled states have been experimentally demonstrated with superconducting qubits based on circuit QED [24-29]. Recently, using SC qubits coupled to a single resonator, a Greenberger-Horne-Zeilinger entangled state of ten SC qubits has been demonstrated in experiments [30]. With solid-state platforms, this is the largest number of entangled qubits reported so far, after the previous experimental demonstration of entanglement with five superconducting qubits via capacitance coupling was reported [31].

A significant experimental challenge for entanglement engineering is how to create quantum entanglement with high-dimensional quantum systems. Compared with a qubit (two-level system), a qutrit (three-level system) has a larger Hilbert space and thus can be used to encode more information in QIP and communication. As is well known, high-dimensional (HD) quantum entanglement is not only of great interest in providing an additional way for the fundamental tests of quantum nonlocality, but also central for HD-based quantum computation, quantum communication, quantum error correction, and quantum simulation. Over the past years, base on cavity or circuit QED, a large number of theoretical methods have been presented for creating two-qubit or multi-qubit entangled states with various physical systems (e.g., atoms, nitrogen-vacancy centers, quantum dots, and SC devices) [10-12,1823,32-39]. However, after a deep search of the literature, we found that based on cavity QED or circuit QED, how to create entangled states of qutrits or higher-dimensional quantum systems with natural or artificial atoms has been rarely investigated.

*Electronic address: yangcp@hznu.edu.cn 


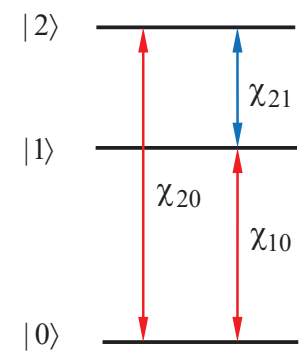

FIG. 1: Level diagram of a transmon qutrit with the ladder-type three lowest levels. For a transmon qutrit, the level spacing between the upper two levels is smaller than that between the two lowest levels. In addition, for a transtion qutrit, the transition between the two levels $|0\rangle$ and $|2\rangle$ is much weaker compared to the transition between the upper two levels and the transition between the two lowest levels [34]. Namely, $\chi_{20} \ll \chi_{10}, \chi_{21}$. Here, $\chi_{i j}$ is the transition matrix element for the two levels $|j\rangle$ and $|i\rangle(i j=20,10,21)$.

Motivated by the above, in this work we will consider a physical system, which consists of two superconducting transmon qutrits coupled to a single microwave cavity or resonator. Note that transmon devices have been considered as one of the best SC information carriers because of their relatively longer coherence times compared with other type of SC devices (e.g., flux, phase, and charge qubits/qutrits, etc.). In the following, we will propose an approach to prepare the two transmon qutrits in an entangled state (either a partially-entangled state or a maximally-entangled state). To the best of our knowledge, our proposal is the first one to show how to create entangled states of qutrits with natural or artificial atoms based on cavity or circuit QED.

As shown below, this proposal has the following advantages: (i) Because only qutrit-cavity resonant interaction and qutrit-pulse resonant interaction are employed, the entanglement creation can be completed within a short time; (ii) There is no requirement on the identical qutrit-cavity coupling constants; thus either non-uniformity in the qutrit device parameters (resulting in nonidentical qutrit level spacings) or non-exact placement of qutrits in the cavity is allowed by this proposal; (iii) Since only a single microwave cavity is used and no auxiliary qutrit is needed, the setup is simple; (iv) There is no need of measurement on the qutrit states or the cavity state, thus the entangled states can be created deterministically; and (v) The degree of entanglement for the prepared entangled state is adjustable by varying the weight factors of the initial state of one qutrit, thus this proposal can be used to create the twoqutrit entangled state varying from a partially entangled state to a maximally entangled state. This proposal is quite general, which can be extended to create a two-qutrit entangled state with a wide range of physical systems, such as two natural atoms (with a ladder-type three-level structure) coupled to an optical cavity, or two artificial atoms of a ladder-type three-level structure (e.g., superconducting phase qutrits, Xmon qutrits, nitrogen-vacancy centers, quantum dots, etc.) coupled to a microwave cavity or resonator.

This paper is organized as follows. In Sec. II, we review the basic theory of a transmon qutrit resonantly interacting with a single cavity or a classical microwave pulse. In Sec. III, we explicitly show how to prepare two transmon qutrits in an entangled state based on circuit QED. In Sec. IV, we give a brief discussion on the experimental feasibility. A concluding summary is given in Sec. V.

\section{BASIC THEORY}

A transmon qutrit has a ladder-type three-level structure [40]. In this work, we consider the three lowest levels of a transmon qutrit, which are denoted as $|0\rangle,|1\rangle$ and $|2\rangle$ (Fig.1). For a transmon qutrit, the transition between the two levels $|0\rangle$ and $|2\rangle$ is much weaker compared to the transition between the two levels $|0\rangle$ and $|1\rangle$ and the transition between the two levels $|1\rangle$ and $|2\rangle$ [40]. As shown in next section, the entanglement generation requires two types of resonant interaction. Namely, the resonant interaction between a cavity and the $|0\rangle \leftrightarrow|1\rangle$ transition of the qutrit; and the resonant interaction between a classical pulse and the $|1\rangle \leftrightarrow|2\rangle$ transition of the qutrit. To make our presentation given in next section clear, in the following we will give a brief review on the state evolutions for these two kinds of interactions.

\section{A. Qutrit coupled to a single cavity}

Consider a transmon qutrit coupled to a single-mode cavity. Assume that the cavity is resonantly coupled to the transition between the two lowest levels $|0\rangle$ and $|1\rangle$ while highly detunned (decoupled) from the transitions between other levels of the qutrit. Under this consideration, the interaction Hamiltonian in the interaction picture, after making the rotating-wave approximation, can be written as $H_{\mathrm{I}_{1}}=\hbar g a^{\dagger}|0\rangle\langle 1|+$ h.c., where $a^{\dagger}(a)$ is the creation 
(annihilation) operator of the cavity and $g$ is the coupling constant between the cavity and the $|0\rangle \leftrightarrow|1\rangle$ transition of the qutrit.

Under the Hamiltonian $H_{\mathrm{I}_{1}}$, one can obtain the following state evolution:

$$
\begin{aligned}
& |0\rangle|0\rangle_{c} \rightarrow|0\rangle|0\rangle_{c} \\
& |1\rangle|0\rangle_{c} \rightarrow-i \sin g t|0\rangle|1\rangle_{c}+\cos g t|1\rangle|0\rangle_{c}, \\
& |0\rangle|1\rangle_{c} \rightarrow \cos g t|0\rangle|1\rangle_{c}-i \sin g t|1\rangle|0\rangle_{c},
\end{aligned}
$$

where $|0\rangle_{c}$ is the vacuum state of the cavity while $|1\rangle_{c}$ is the single-photon state of the cavity.

As shown in next section, the entanglement preparation requires that a cavity interacts with each of two qutrits 1 and 2. In reality, it is a challenge to have the qutrit-cavity coupling constants to be identical for both of the qutrits. Thus, we replace the coupling constant $g$ with $g_{1}$ for qutrit 1 while $g_{2}$ for qutrit 2 .

\section{B. Qutrit driven by a classical pulse}

Let us consider a transmon qutrit driven by a microwave pulse. If the pulse is resonantly coupled to the $|0\rangle \leftrightarrow$ |1 transition but highly detunned (decoupled) from the transitions between other levels of the qutrit. Then, the interaction Hamiltonian in the interaction picture is given by $H_{\mathrm{I}_{2}}=\hbar\left(\Omega_{10} e^{i \phi}|0\rangle\langle 1|+\right.$ h.c. $)$, where $\phi$ is the initial phase of the pulse while $\Omega_{10}$ is the Rabi frequency of the pulse. From the Hamiltonian $H_{\mathrm{I}_{2}}$, it is easy to show that a pulse of duration $t$ results in the following state evolution:

$$
\begin{aligned}
& |0\rangle \rightarrow \cos \Omega_{10} t|0\rangle-i e^{-i \phi} \sin \Omega_{10} t|1\rangle, \\
& |1\rangle \rightarrow-i e^{i \phi} \sin \Omega_{10} t|0\rangle+\cos \Omega_{10} t|1\rangle .
\end{aligned}
$$

Similarly, when the pulse is resonantly coupled to the $|1\rangle \leftrightarrow|2\rangle$ transition but highly detunned (decoupled) from the transitions between other levels of the qutrit, the interaction Hamiltonian in the interaction picture is given by $H_{\mathrm{I}_{3}}=\hbar\left(\Omega_{21} e^{i \phi}|1\rangle\langle 2|+\right.$ h.c. $)$, where $\Omega_{21}$ is the Rabi frequency of the pulse. Based on this Hamiltonian, we can obtain the following state rotation:

$$
\begin{aligned}
& |1\rangle \rightarrow \cos \Omega_{21} t|1\rangle-i e^{-i \phi} \sin \Omega_{21} t|2\rangle, \\
& |2\rangle \rightarrow-i e^{i \phi} \sin \Omega_{21} t|1\rangle+\cos \Omega_{21} t|2\rangle .
\end{aligned}
$$

Finally, it should be mentioned that when the cavity is highly detunned (decoupled) from the $|1\rangle \leftrightarrow|2\rangle$ and $|0\rangle \leftrightarrow|2\rangle$ transitions of the qutrit, there is neither coupling between the cavity and the qutrit nor transition between the levels $|2\rangle$ and $|1\rangle$ or $|0\rangle$ induced by the cavity. As a result, the states $|2\rangle|0\rangle_{c}$, and $|2\rangle|1\rangle_{c}$ remain unchanged during the cavity on resonance with the $|0\rangle \leftrightarrow|1\rangle$ transition of the qutrit. For a similar reason, the state $|2\rangle(|0\rangle)$ of the qutrit is unaffected during applying the pulse resonant with the $|0\rangle \leftrightarrow|1\rangle(|1\rangle \leftrightarrow|2\rangle)$ transition of the qutrit. These results, togeter with the results given in Eqs. (1-3), will be employed for the entanglement generation, as shown in the next section.

\section{GENERATION OF TWO-TRANSMON-QUTRIT ENTANGLED STATES}

Let us now consider two transmon qutrits 1 and 2 embedded in a single-mode cavity. Initially, the two qutrits are decoupled from the cavity, qutrit 1 is in the state $\alpha|0\rangle+\gamma|1\rangle+\beta|2\rangle$, qutrit 2 is in the ground state $|0\rangle$, and the cavity is in the vacuum state $|0\rangle_{c}$. The initial state of qutrit 1 here can be prepared by applying classical microwave pulses. The initial state of the whole system is thus given by $|\psi\rangle_{\text {in }}=\left(\alpha|0\rangle_{1}+\gamma|1\rangle_{1}+\beta|2\rangle_{1}\right)|0\rangle_{2}|0\rangle_{c}$. Hereafter, the subscripts 1 and 2 represent qutrits 1 and 2 , respectively.

To begin with, we define $\omega_{20}\left(\omega_{21}\right)$ as the $|0\rangle \leftrightarrow|1\rangle(|1\rangle \leftrightarrow|2\rangle)$ transition frequency of each qutrit and $\Omega_{10}\left(\Omega_{21}\right)$ as the pulse Rabi frequency of the coherent $|0\rangle \leftrightarrow|1\rangle(|1\rangle \leftrightarrow|2\rangle)$ transition. In addition, the frequency, initial phase, and duration of a microwave pulse are denoted as $\left\{\omega, \phi, t^{\prime}\right\}$ in the remaining of the paper.

The operations for creating the entangled state (3) are described below:

Step 1: Apply a microwave pulse of $\left\{\omega_{10},-\pi / 2, \pi /\left(4 \Omega_{10}\right)\right\}$ and then a microwave pulse of $\left\{\omega_{21},-\pi / 2, \pi /\left(2 \Omega_{21}\right)\right\}$ to qutrit 2 [Fig. 2(a)]. According to Eqs. (2) and (3), the pulse application results in the state transformation $|0\rangle_{2} \rightarrow$ $\left(|0\rangle_{2}+|2\rangle_{2}\right) / \sqrt{2}$ (see Appendix). Therefore, the initial state $|\psi\rangle_{\text {in }}$ of the system becomes

$$
\frac{1}{\sqrt{2}}\left(\alpha|0\rangle_{1}+\gamma|1\rangle_{1}+\beta|2\rangle_{1}\right)\left(|0\rangle_{2}+|2\rangle_{2}\right)|0\rangle_{c} .
$$




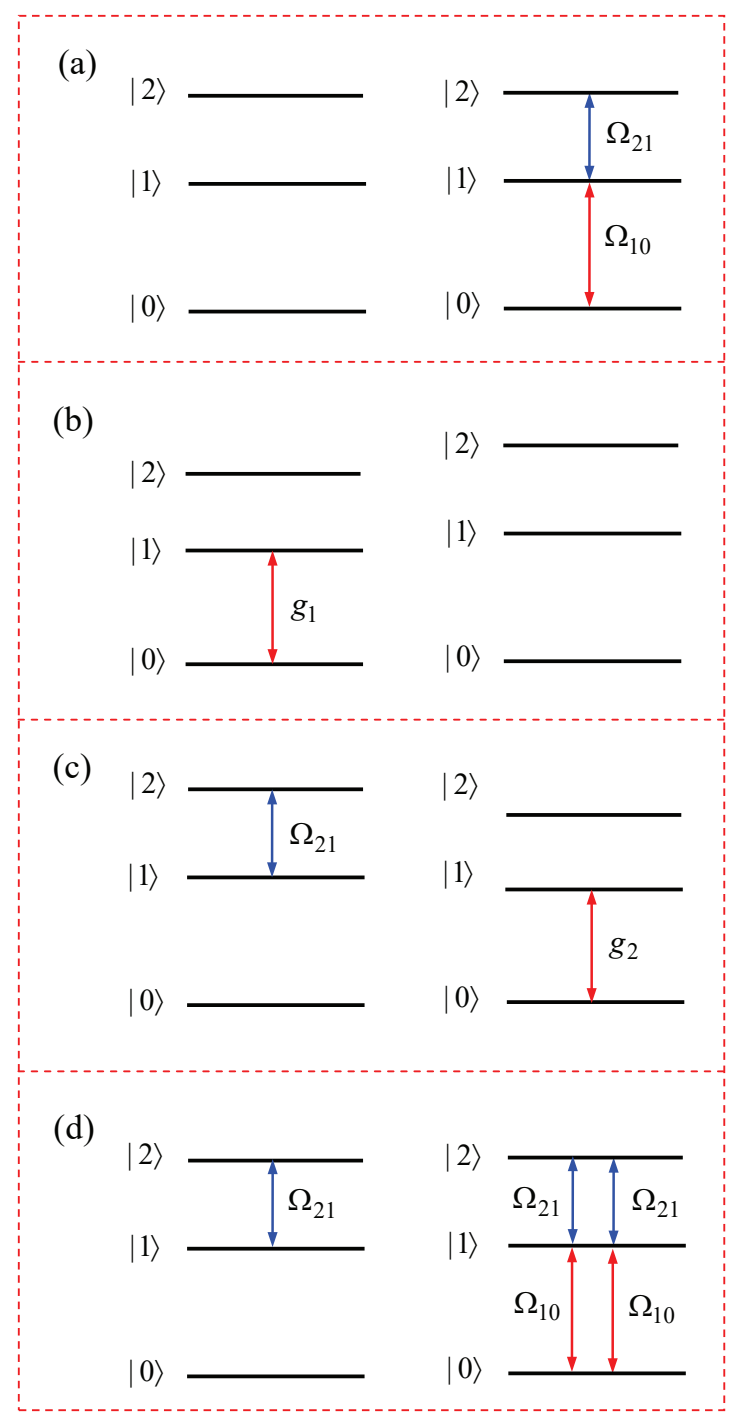

FIG. 2: (Color online) Illustration for the change of the level structure of two transmon qutrits during the entanglement preparation. In (a), (b), (c), and (d), figures on the left represent the level structure for qutrit 1 while figures on the right represent the level structure for qutrit 2. (a) Decoupling of qutrits 1 and 2 from the cavity during applying microwave pulses to qutrit 2. The pulse with a Rabi frequency $\Omega_{21}$ is applied after the pulse of a Rabi frequency $\Omega_{10}$ is completed. (b) Decoupling of qutrit 2 from the cavity during bringing the $|0\rangle \leftrightarrow|1\rangle$ transition of qutrit 1 to resonance with the cavity. $g_{1}$ is the coupling constant. (c) Decoupling of qutrit 1 from the cavity during applying a microwave pulse to qutrit 1 and bringing the $|0\rangle \leftrightarrow|1\rangle$ transition of qutrit 2 to resonance with the cavity. $g_{2}$ is the coupling constant. (d) Decoupling of qutrits 1 and 2 from the cavity during applying microwave pulses to each qutrit (for the sequence of the applied pulses, see the operation of step 5 in the text). In (b) [(c)], during the cavity on resonance with the $|0\rangle \leftrightarrow|1\rangle$ transition of qutrit 1 (2), the cavity is highly detuned (or decoupled) from the $|1\rangle \leftrightarrow|2\rangle$ transition of qutrit 1 (2) can be made by having the $|1\rangle \leftrightarrow|2\rangle$ transition frequency highly detuning from the cavity frequency. Note that the coupling or decoupling of the qutrits with the cavity can be achieved by adjusting the level spacings of the qutrits [40-43]. 
Step 2: Bring the $|0\rangle \leftrightarrow|1\rangle$ transition of qutrit 1 to resonance with the cavity for an interaction time $\pi /\left(2 g_{1}\right)$ [Fig. 2(b)]. According to Eq. (1), we have $|1\rangle_{1}|0\rangle_{c} \rightarrow-i|0\rangle_{1}|1\rangle_{c_{\backslash}}$. Thus, the state (4) changes to

$$
\frac{1}{\sqrt{2}}\left[\left(\alpha|0\rangle_{1}+\beta|2\rangle_{1}\right)\left(|0\rangle_{2}+|2\rangle_{2}\right)|0\rangle_{c}-i \gamma|0\rangle_{1}\left(|0\rangle_{2}+|2\rangle_{2}\right)|1\rangle_{c}\right]
$$

Step 3: Bring the $|0\rangle \leftrightarrow|1\rangle$ transition of qutrit 2 to resonance with the cavity for an interaction time $\pi / g_{2}$ [Fig. 2(c)] resulting in $|0\rangle_{2}|1\rangle_{c} \rightarrow-|0\rangle_{2}|1\rangle_{c}$ according to Eq. (1). The state (5) thus changes to

$$
\frac{1}{\sqrt{2}}\left[\left(\alpha|0\rangle_{1}+\beta|2\rangle_{1}\right)\left(|0\rangle_{2}+|2\rangle_{2}\right)|0\rangle_{c}-i \gamma|0\rangle_{1}\left(-|0\rangle_{2}+|2\rangle_{2}\right)|1\rangle_{c}\right] .
$$

Step 4: Bring the $|0\rangle \leftrightarrow|1\rangle$ transition of qutrit 1 to resonance with the cavity for an interaction time $3 \pi /\left(2 g_{1}\right)$ [Fig. 2(b)]. According to Eq. (1), we have $|0\rangle_{1}|1\rangle_{c} \rightarrow i|1\rangle_{1}|0\rangle_{c}$. Hence, the state (6) changes to

$$
\frac{1}{\sqrt{2}}\left[\left(\alpha|0\rangle_{1}+\beta|2\rangle_{1}\right)\left(|0\rangle_{2}+|2\rangle_{2}\right)+\gamma|1\rangle_{1}\left(-|0\rangle_{2}+|2\rangle_{2}\right)\right]|0\rangle_{c} .
$$

Step 5: Apply microwave pulses of $\left\{\omega_{10},-\pi / 2, \pi /\left(2 \Omega_{10}\right)\right\},\left\{\omega_{21}, \pi / 2, \pi /\left(4 \Omega_{21}\right)\right\},\left\{\omega_{10}, \pi / 2,3 \pi /\left(4 \Omega_{10}\right)\right\}$, and then $\left\{\omega_{21}, \pi / 2, \pi /\left(2 \Omega_{21}\right)\right\}$ to qutrit 2 in turn [Fig. 2(d)]. According to Eqs. (2) and (3), the pulse application leads to $\left(-|0\rangle_{2}+|2\rangle_{2}\right) / \sqrt{2} \rightarrow|1\rangle_{2}$ but nothing to the state $\left(|0\rangle_{2}+|2\rangle_{2}\right) / \sqrt{2}$ of qutrit 2 (see Appendix). Meanwhile, apply a microwave pulse of $\left\{\omega_{21}, \pi / 2, \pi /\left(2 \Omega_{21}\right)\right\}$ to qutrit 1 [Fig. 2(d)], resulting in $|2\rangle_{1} \rightarrow|1\rangle_{1}$ and $|1\rangle_{1} \rightarrow-|2\rangle_{1}$ according to Eq. (3). Therefore, the state (7) becomes

$$
\left[\frac{1}{\sqrt{2}} \alpha\left(|0\rangle_{1}+\beta|1\rangle_{1}\right)\left(|0\rangle_{2}+|2\rangle_{2}\right)-\gamma|2\rangle_{1}|1\rangle_{2}\right]|0\rangle_{c} .
$$

Step 6: Bring the $|0\rangle \leftrightarrow|1\rangle$ transition of qutrit 1 to resonance with the cavity for an interaction time $\pi /\left(2 g_{1}\right)$ [Fig. 2(b)]. As a result, we have $|1\rangle_{1}|0\rangle_{c} \rightarrow-i|0\rangle_{1}|1\rangle_{c}$. Thus, the state (8) becomes

$$
\frac{1}{\sqrt{2}}\left[\alpha|0\rangle_{1}\left(|0\rangle_{2}+|2\rangle_{2}\right)|0\rangle_{c}-i \beta|0\rangle_{1}\left(|0\rangle_{2}+|2\rangle_{2}\right)|1\rangle_{c}\right]-\gamma|2\rangle_{1}|1\rangle_{2}|0\rangle_{c} .
$$

Step 7: Bring the $|0\rangle \leftrightarrow|1\rangle$ transition of qutrit 2 to resonance with the cavity for an interaction time $\pi / g_{2}$ [Fig. 2(c)], resulting in $|0\rangle_{2}|1\rangle_{c} \rightarrow-|0\rangle_{2}|1\rangle_{c}$ and $|1\rangle_{2}|0\rangle_{c} \rightarrow-|1\rangle_{2}|0\rangle_{c}$. Meanwhile, apply a microwave pulse of $\left\{\omega_{21}, \pi / 2, \pi / \Omega_{21}\right\}$ to qutrit 1 [Fig. 2(c)], resulting in $|2\rangle_{1} \rightarrow-|2\rangle_{1}$. Thus, the state (9) becomes

$$
\frac{1}{\sqrt{2}}\left[\alpha|0\rangle_{1}\left(|0\rangle_{2}+|2\rangle_{2}\right)|0\rangle_{c}-i \beta|0\rangle_{1}\left(-|0\rangle_{2}+|2\rangle_{2}\right)|1\rangle_{c}\right]-\gamma|2\rangle_{1}|1\rangle_{2}|0\rangle_{c}
$$

Step 8: Bring the $|0\rangle \leftrightarrow|1\rangle$ transition of qutrit 1 to resonance with the cavity for an interaction time $3 \pi /\left(2 g_{1}\right)$ [Fig. 2(b)]. As a result, we have $|0\rangle_{1}|1\rangle_{c} \rightarrow i|1\rangle_{1}|0\rangle_{c}$. Therefore, the state (10) changes to

$$
\left\{\frac{1}{\sqrt{2}}\left[\alpha|0\rangle_{1}\left(|0\rangle_{2}+|2\rangle_{2}\right)+\beta|1\rangle_{1}\left(-|0\rangle_{2}+|2\rangle_{2}\right)\right]-\gamma|2\rangle_{1}|1\rangle_{2}\right\}|0\rangle_{c} .
$$

Step 9: Apply a microwave pulse of $\left\{\omega_{21}, \pi / 2, \pi /\left(2 \Omega_{21}\right)\right\}$ and then a microwave pulse of $\left\{\omega_{10}, \pi / 2, \pi /\left(4 \Omega_{10}\right)\right\}$ to qutrit 2 [Fig. 2(a)], resulting in the state transformations $\left(|0\rangle_{2}+|2\rangle_{2}\right) / \sqrt{2} \rightarrow|0\rangle_{2},\left(-|0\rangle_{2}+|2\rangle_{2}\right) / \sqrt{2} \rightarrow|1\rangle_{2}$, and $|1\rangle_{2} \rightarrow-|2\rangle_{2}$ (see Appendix). Hence, the state (11) becomes

$$
\left(\alpha|0\rangle_{1}|0\rangle_{2}+\beta|1\rangle_{1}|1\rangle_{2}+\gamma|2\rangle_{1}|2\rangle_{2}\right)|0\rangle_{c} .
$$

The above operations are illustrated in Fig. 3. The result (12) shows that the two qutrits are prepared in an entangled state

$$
|\psi\rangle_{\mathrm{ent}}=\alpha|0\rangle_{1}|0\rangle_{2}+\beta|1\rangle_{1}|1\rangle_{2}+\gamma|2\rangle_{1}|2\rangle_{2},
$$




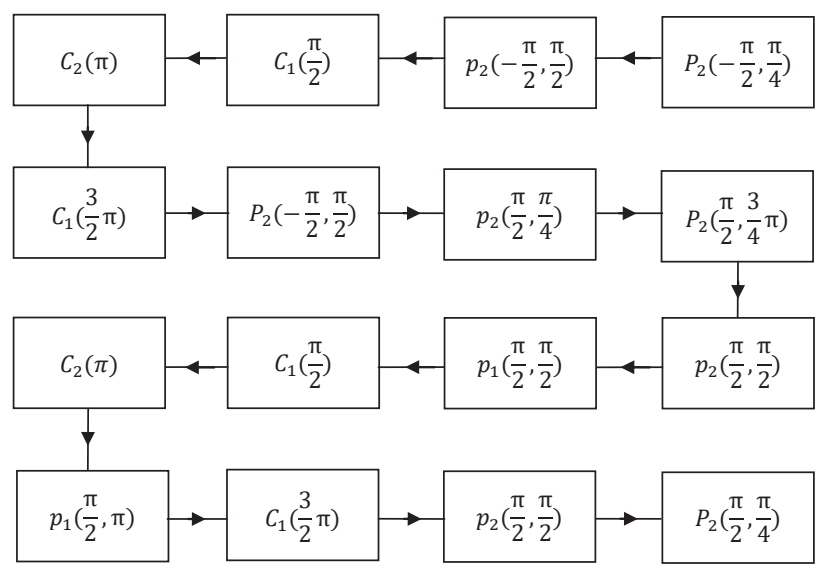

FIG. 3: (Color online) Circuit diagram for the two-qutrit entanglement preparation (the operation sequence follows the arrows). The subscript $j$ of $C_{j}, p_{j}$, and $P_{j}$ represents qutrit $j(j=1,2)$ on which the operation is performed. $C_{1}\left(g_{1} t\right)$, with $g_{1} t=\pi / 2$ or $3 \pi / 2$, represents the operation that the cavity resonantly interacts with the $|0\rangle \leftrightarrow|1\rangle$ transition of qutrit 1 . $C_{2}\left(g_{2} t\right)$, with $g_{2} t=\pi$, represents the operation that the cavity resonantly interacts with the $|0\rangle \leftrightarrow|1\rangle$ transition of qutrit 2 . Here $g_{1}$ or $g_{2}$ is the qutrit-cavity coupling constant, while $t$ is the qutrit-cavity interaction time. In addition, $p_{j}\left(\phi, \Omega_{21} t\right)$, with $\phi=-\pi / 2$ or $\pi / 2$ and $\Omega_{21} t=\pi / 4, \pi / 4$ or $\pi$, represents the operation that a microwave pulse is resonant with the $|1\rangle \leftrightarrow|2\rangle$ transition of qutrit $j(j=1,2)$; while $P_{j}\left(\phi, \Omega_{10} t\right)$, with $\phi=-\pi / 2$ or $\pi / 2$ and $\Omega_{10} t=\pi / 4, \pi / 2$ or $3 \pi / 4$, represents the operation that a microwave pulse is resonant with the $|0\rangle \leftrightarrow|1\rangle$ transition of qutrit $j(j=2)$. Here, $\phi$ is the pulse initial phase, $\Omega_{10}$ or $\Omega_{21}$ is the pulse Rabi frequency, while $t$ is the pulse duration.

while the cavity returns to its original vacuum state after the entire operation. It can be seen that the prepared entangled state (13) is different from the two-qubit entangled states $\alpha|00\rangle+\beta|11\rangle / \sqrt{2}$ and $\alpha|01\rangle+\beta|10\rangle$. It is well known that the two-qubit entangled states have been experimentally generated with various physical systems. Interestingly, it is noted that the procedure for entangling the two qutrits has nothing to do with $\alpha, \beta$, and $\gamma$. Moreover, the degree of entanglement for the prepared two-qutrit entangled state (13) is controllable by varying the weight factors $\alpha, \beta$, and $\gamma$ involved in the initial state of qutrit 1. For $\alpha=\beta=\gamma=1 / \sqrt{3}$, the prepared entangled state (13) is a maximally entangled state $\left(|0\rangle_{1}|0\rangle_{2}+|1\rangle_{1}|1\rangle_{2}+|2\rangle_{1}|2\rangle_{2}\right) / \sqrt{3}$ of the two qutrits. Otherwise, the two qutrits are prepared in a partially entangled state.

From description given above, one can see that this proposal does not require that the qutrit-cavity coupling constants $g_{1}$ and $g_{2}$ are identical. Thus, either non-uniformity in the qutrit device parameters or non-exact placement of qutrits in the cavity is allowed, which significantly reduces the experiemental engineering difficulty. In addition, neither auxiliary qutrit nor measurement is needed for the two-qutrit entanglement production. Furthermore, as shown above, this proposal only requires two different microwave-pulse frequencies $\left(\omega_{10}\right.$ and $\left.\omega_{21}\right)$, which are readily achievable in current experiments [41].

Before ending this section, several points are addressed as follows:

(a) During the operations of steps (2-4) and (6-8), the $|0\rangle \leftrightarrow|1\rangle$ transition of qutrit 1 or 2 is on resonance with the cavity. This can be achieved by adjusting the level spacings of the qutrits. For superconducting qutrits, the level spacings can be rapidly (within a few nanoseconds) adjusted by varying external control parameters (e.g., the magnetic flux applied to a superconducting loop of phase, transmon, Xmon, or flux qubits; see, e.g., [42-45]).

(b). Durng the cavity interacting with a qutrit, the other qutrit is decoupled from the cavity. During the pulse application, both qutrits are decoupled from the cavity, in order to avoid the effect of the unwanted qutrit-cavity interactions. In addition, during the cavity on resonance with the $|0\rangle \leftrightarrow|1\rangle$ transition of a qutrit, the $|1\rangle \leftrightarrow|2\rangle$ transition of this qutrit is highly detuned (or decoupled) from the cavity. In principle, these conditions can be satisfied by adjusting the level spacings of the qutrits [42-45].

(c). The ladder-type level structure is available in natural atoms or other artificial atoms such as nitrogen-vacancy (NV) centers, quantum dots, and various other SC qutrits (SC phase, charge, and Xmon qubits). For NV centers, the level spacings can be readily adjusted by changing the external magnetic field applied along the crystalline axis of each NV center $[46,47]$. For quantum dots and atoms, the level spacings can be adjusted by changing the voltage on the electrodes around each atom/quantum dot [48]. Thus, this proposal can be applied to create the two-qutrit entangled state (13) in a wide range of physical systems.

(d). The method presented here is applicable to a $1 \mathrm{D}, 2 \mathrm{D}$, or $3 \mathrm{D}$ cavity or resonator as long as the conditions described above can be met. 


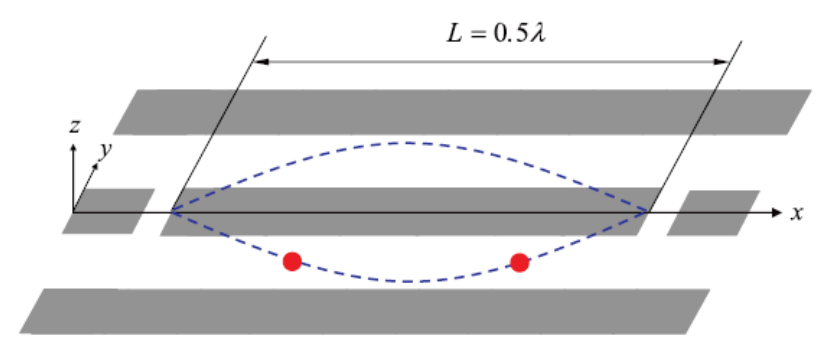

FIG. 4: (Color online) Setup for two transmon qutrits (red dots) embedded in a one-dimensional coplanar waveguide resonator. $\lambda$ is the wavelength of the resonator mode, and $L$ is the length of the resonator. The two (blue) curved lines represent the standing wave magnetic field of the resonator in the $z$ direction, which is perpendicular to the surface of the superconducting loop of each transmon qutrit. The two transmon qutrits are placed at locations where the magnetic fields are the same to achieve an identical coupling strength for each qutrit. The superconducting loop (not shown) of each transmon qutrit is located in the plane of the resonator between the two lateral ground planes (i.e., the x-y plane).

\section{DISCUSSION AND POSSIBLE EXPERIMENTAL IMPLEMENTATION}

The total operation time is

$$
\tau=\frac{7 \pi}{4 \Omega_{10}}+\frac{13 \pi}{4 \Omega_{21}}+\frac{4 \pi}{g_{1}}+\frac{2 \pi}{g_{2}}+8 \tau_{d}
$$

where $t_{d}$ is the typical time required for adjusting the qutrit level spacings. To reduce the effect of decoherence from the qutrits, the operation time $\tau$ should be much smaller than the energy relaxation time and the dephasing time of qutrits. In addition, to reduce the effect of dissipation from the cavity, $\tau$ should be much smaller than the lifetime of the cavity mode, which is given by $\kappa^{-1}=Q /\left(2 \pi \nu_{c}\right)\left(Q\right.$ is the quality factor of the cavity while $\nu_{c}$ is the cavity frequency). In principle, these requirements can be satisfied for the following reasons. First, the operation time $\tau$ can be shortened by increasing the coupling constants $g_{1}$ and $g_{2}$ (e.g., placing each qutrit at an antinode of the cavity magnetic field), increasing the pulse Rabi frequencies $\Omega_{10}$ and $\Omega_{21}$ (e.g., through increasing the pulse intensity), and rapidly adjusting the qutrit level spacings (e.g., $\tau_{d} \sim 1-3 \mathrm{~ns}$ is the typical time for adjusting the level spacings of a superconducting qutrit in experiments $[41,43,49])$. Second, one can choose qutrits with long decoherence times. Last, $\kappa^{-1}$ can be increased by employing a high- $Q$ resonator.

Let us now consider the experimental possibility of preparing the two-qutrit entangled state (13) with two superconducting transmon qutrits embedded in a one-dimensional transmission line resonator (TLR) (Fig. 4).

When dissipation and dephasing are considered, the dynamics of the lossy system is determined by

$$
\begin{aligned}
\frac{d \rho}{d t}= & -i\left[H_{\mathrm{I}}, \rho\right]+\kappa \mathcal{L}[a] \\
& +\sum_{j=1,2} \gamma_{10, j} \mathcal{L}\left[\sigma_{10, j}^{-}\right]+\gamma_{21, j} \mathcal{L}\left[\sigma_{21, j}^{-}\right]+\gamma_{20, j} \mathcal{L}\left[\sigma_{20, j}^{-}\right] \\
& +\sum_{j=1,2}\left\{\gamma_{\varphi 1, j}\left(\sigma_{11, j} \rho \sigma_{11, j}-\sigma_{11, j} \rho / 2-\rho \sigma_{11, j} / 2\right)\right\} \\
& +\sum_{j=1,2}\left\{\gamma_{\varphi 2, j}\left(\sigma_{22, j} \rho \sigma_{22, j}-\sigma_{22, j} \rho / 2-\rho \sigma_{22, j} / 2\right)\right\}
\end{aligned}
$$

where $H_{\mathrm{I}}$ is the Hamiltonian $H_{\mathrm{I}_{1}}, H_{\mathrm{I}_{2}}$,or $H_{\mathrm{I}_{3}}$ above, the subscript $j$ represents qutrit $j(j=1,2) ; \sigma_{10, j}^{-}=|0\rangle_{j}\langle 1|$, $\sigma_{21, j}^{-}=|1\rangle_{j}\left\langle 2\left|, \sigma_{20, j}^{-}=\right| 0\right\rangle_{j}\left\langle 2\left|, \sigma_{11, j}=\right| 1\right\rangle_{j}\left\langle 1\left|, \sigma_{22, j}=\right| 2\right\rangle_{j}\langle 2| ;$ and $\mathcal{L}[\xi]=\xi \rho \xi^{\dagger}-\xi^{\dagger} \xi \rho / 2-\rho \xi^{\dagger} \xi / 2$, with $\xi=a, \sigma_{10, j}^{-}, \sigma_{21, j}^{-}, \sigma_{20, j}^{-}$. Here, $\kappa$ is the photon decay rate of the cavity or resonator. In addition, $\gamma_{10, j}$ is the energy relaxation rate for the level $|1\rangle$ of qutrit $j, \gamma_{21, j}\left(\gamma_{20, j}\right)$ is the energy relaxation rate of the level $|2\rangle$ of qutrit $j$ for the decay path $|2\rangle \longrightarrow|1\rangle(|0\rangle)$, and $\gamma_{\varphi 1, j}\left(\gamma_{\varphi 2, j}\right)$ is the dephasing rate of the level $|1\rangle(|2\rangle)$ of qutrit $j(j=1,2)$.

The fidelity of the operations is given by

$$
\mathcal{F}=\sqrt{\left\langle\psi_{\text {id }}|\rho| \psi_{\text {id }}\right\rangle}
$$

where $\left|\psi_{\text {id }}\right\rangle$ is the output state of an ideal system without dissipation and dephasing; while $\rho$ is the final practical density operator of the system when the operation is performed in a realistic situation. As an example, consider 


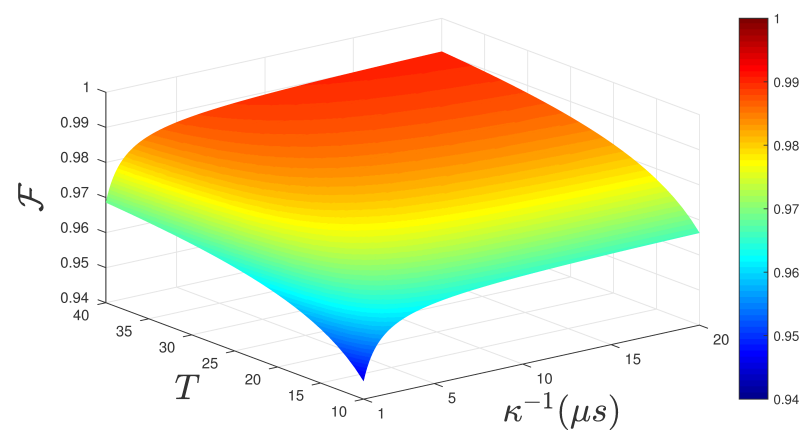

FIG. 5: (Color online) Fidelity versus $T$ and $\kappa^{-1}$. The parameters used in the numerical simulation are referred to the text.

$\alpha=\beta=\gamma=1 / \sqrt{3}$, for which the prepared entangled state (13) is $\left|\psi_{\text {id }}\right\rangle=\left(|0\rangle_{1}|0\rangle_{2}+|1\rangle_{1}|1\rangle_{2}+|2\rangle_{1}|2\rangle_{2}\right) / \sqrt{3}$ for the ideal case, which corresponds to the case that the initial state of the whole system is $|\psi\rangle_{\text {in }}=$ $1 / \sqrt{3}\left(\alpha|0\rangle_{1}+\gamma|1\rangle_{1}+\beta|2\rangle_{1}\right)|0\rangle_{2}|0\rangle_{c}$.

The parameters used in the numerical simulation are: (i) $\gamma_{10, j}^{-1}=2 T \mu \mathrm{s}, \gamma_{20, j}^{-1}=5 T \mu \mathrm{s}, \gamma_{21, j}^{-1}=T \mu \mathrm{s}, \gamma_{\phi 1, j}^{-1}=\gamma_{\phi 2, j}^{-1}=$ $T \mu \mathrm{s}$, (ii) $g_{1}, g_{2}, \Omega_{10}, \Omega_{21}=2 \pi \times 100 \mathrm{MHz}$. The coupling strengths and the pulse Rabi frequencies with the values chosen here are readily available in experiments because a coupling strength $g / 2 \pi \sim 220 \mathrm{MHz}$ has been experimentally demonstrated for a transmon device coupled to a TLR [50] and a microwave pulse Rabi frequency $\Omega / 2 \pi \sim 300 \mathrm{MHz}$ has been reported in experiments [51].

By solving the master equation (15), we numerically calculate the fidelity versus $T$ and $\kappa^{-1}$, as shown in Fig. 5 . Figure 5 illustrates that when $T=30 \mu \mathrm{s}$ and $\kappa^{-1} \geq 2.5 \mu \mathrm{s}$, fidelity exceeds $98.03 \%$. This result implies that a high fidelity can be obtained when the dissipation and dephasing are taken into account.

For $T=30 \mu \mathrm{s}$, we have $\gamma_{10, j}^{-1}=60 \mu \mathrm{s}, \gamma_{20, j}^{-1}=150 \mu \mathrm{s}, \gamma_{21, j}^{-1}=30 \mu \mathrm{s}$, and $\gamma_{\phi 1, j}^{-1}=\gamma_{\phi 2, j}^{-1}=30 \mu \mathrm{s}$, which are much longer than the total operation time $\sim 67$ ns (estimated based on the coupling constants and the pulse Rabi frequencies chosen above and for $\tau_{d} \sim 1.5 \mathrm{~ns}$ ). Note that energy relaxation time and dephasing time can be made to be on the order of $25-100 \mu \mathrm{s}$ for state-of-the-art superconducting transmon devices at the present time [52-55]. It is worth noting that for an ideal transmon, the $|0\rangle \leftrightarrow|2\rangle$ transition is theoretically zero due to the selection rule [40,56]; and in practice, there exists a weak transition between the two levels $|0\rangle$ and $|2\rangle[57]$. Thus, $\gamma_{20, j}^{-1} \gg \gamma_{10, j}^{-1}, \gamma_{21, j}^{-1}$.

For a transmon qutrit, the typical transition frequency between the two lowest levels $|0\rangle$ and $|1\rangle$ can be made to be $3-10 \mathrm{GHz}$. Thus, as an example, choose $\nu_{c} \sim 3 \mathrm{GHz}$. For $\kappa^{-1}=2.5 \mu \mathrm{s}$, we have $Q \sim 4.7 \times 10^{4}$. The required resonator quality factor here is achievable in experiments because TLRs with a (loaded) quality factor $Q \sim 10^{6}$ have been experimentally demonstrated [58,59].

The analysis presented above shows that high-fidelity generation of the two-qutrit entangled state is feasible within present-day circuit QED.

Finally, for two superconducting qutrits located in a microwave resonator, they can be well separated, because the dimension of a superconducting qutrit is 10 to 100 micrometers while the wavelength of the cavity mode for a microwave superconducting resonator is 1 to a few centimeters [60,61]. As long as the two qutrits are well separated in space (Fig. 4), the loop current of one qutrit affecting the other qutrit and the direct coupling between the two qutrits are negligible, which can be reached by designing the qutrits and the resonator appropriately.

\section{CONCLUSION}

We have presented a method to entangle two transmon qutrits based on circuit QED. Since only resonant interactions are employed, the entanglement can be produced within a short time (a few tens of nanoseconds) and thus decoherence from the qutrits and the cavity are greatly suppressed. As shown above, this proposal does not require measurement on the states of the qutrits or the cavity-mode photons and only requires resonant qutrit-cavity interaction and resonant qutrit-pulse interaction for each step of the operation. Thus, this proposal is easy to implement in experiments. This proposal is quite general and can be applied to accomplish the same task with a wide range of physical systems. To the best of our knowledge, our work is the first to show how to create entangled states of qutrits with natural or artificial atoms based on cavity or circuit QED. We remark that the number of pulses can be reduced by employing dispersive qutrit-cavity interaction but the operation time will become much prolonged due to the large detuning technique to be used. We hope that this work will stimulate the experimental activities in the near future.

\section{ACKNOWLEDGMENTS}


This work was supported in part by the Natural Science Foundation of Fujian Province of China under Grant No. 2015J01020, the Zhejiang Natural Science Foundation under Grant No. LZ13A040002, the NKRDP of China (Grant No. 2016YFA0301802), and the National Natural Science Foundation of China under Grant Nos. [11074062, 11374083, 11774076].

\section{APPENDIX}

We here give a derivation on the state transformations induced by the application of the pulses, for the operations of steps 1,5 , and 9 described above.

For step 1: A microwave pulse of $\left\{\omega_{10},-\pi / 2, \pi /\left(4 \Omega_{10}\right)\right\}$ and then a microwave pulse of $\left\{\omega_{21},-\pi / 2, \pi /\left(2 \Omega_{21}\right)\right\}$ were applied to qutrit 2 [Fig. 2(a)]. The first pulse results in $|0\rangle_{2} \rightarrow\left(|0\rangle_{2}+|1\rangle_{2}\right) / \sqrt{2}$ according to Eq. (2), while the second pulse leads to $|1\rangle_{2} \rightarrow|2\rangle_{2}$ according to Eq. (3). After the two pulses, we thus have the state transformations:

$$
|0\rangle_{2} \stackrel{p 1}{\rightarrow}\left(|0\rangle_{2}+|1\rangle_{2}\right) / \sqrt{2} \stackrel{p 2}{\rightarrow}\left(|0\rangle_{2}+|2\rangle_{2}\right) / \sqrt{2},
$$

where the first transformation is obtained after applying the first pulse while the second transformation is achieved after applying the second pulse. Note that the state $|0\rangle_{2}$ remains unchanged during the second pulse because the pulse is highly detuned (or decoupled) from the $|1\rangle_{2} \rightarrow|2\rangle_{2}$ transition of qutrit 2 .

For step 5: The microwave pulses of $\left\{\omega_{10},-\pi / 2, \pi /\left(2 \Omega_{10}\right)\right\},\left\{\omega_{21}, \pi / 2, \pi /\left(4 \Omega_{21}\right)\right\},\left\{\omega_{10}, \pi / 2,3 \pi /\left(4 \Omega_{10}\right)\right\}$, and then $\left\{\omega_{21}, \pi / 2, \pi /\left(2 \Omega_{21}\right)\right\}$ were applied to qutrit 2 in turn [Fig. 2(d)]. According to Eqs. (2) and (3), the first pulse results in $|0\rangle_{2} \rightarrow|1\rangle_{2}$, the second pulse results in $\left(|1\rangle_{2}+|2\rangle_{2}\right) / \sqrt{2} \rightarrow|1\rangle_{2}$ and $\left(-|1\rangle_{2}+|2\rangle_{2}\right) / \sqrt{2} \rightarrow|2\rangle_{2}$, the third pulse results in $|1\rangle_{2} \rightarrow\left(|0\rangle_{2}-|1\rangle_{2}\right) / \sqrt{2}$ but nothing to the state $|2\rangle_{2}$, and the last pulse leads to $|1\rangle_{2} \rightarrow-|2\rangle_{2}$ and $|2\rangle_{2} \rightarrow|1\rangle_{2}$. Based on these results, we can obtain the following state transformations:

$$
\begin{aligned}
& \left(|0\rangle_{2}+|2\rangle_{2}\right) / \sqrt{2} \stackrel{p 1}{\rightarrow}\left(|1\rangle_{2}+|2\rangle_{2}\right) / \sqrt{2} \stackrel{p 2}{\rightarrow}|1\rangle_{2} \stackrel{p 3}{\rightarrow}\left(|0\rangle_{2}-|1\rangle_{2}\right) / \sqrt{2} \stackrel{p 4}{\rightarrow}\left(|0\rangle_{2}+|2\rangle_{2}\right) / \sqrt{2}, \\
& \left(-|0\rangle_{2}+|2\rangle_{2}\right) / \sqrt{2} \stackrel{p 1}{\rightarrow}\left(-|1\rangle_{2}+|2\rangle_{2}\right) / \sqrt{2} \stackrel{p 2}{\rightarrow}|2\rangle_{2} \stackrel{p 3}{\rightarrow}|2\rangle_{2} \stackrel{p 4}{\rightarrow}|1\rangle_{2},
\end{aligned}
$$

where the first, second, third, and last transformations are obtained after applying the first, second, third, and the last pulses, respectively.

For step 9: A microwave pulse of $\left\{\omega_{21}, \pi / 2, \pi /\left(2 \Omega_{21}\right)\right\}$ and then a microwave pulse of $\left\{\omega_{10}, \pi / 2, \pi /\left(4 \Omega_{10}\right)\right\}$ were applied to qutrit 2 [Fig. 2(a)]. The first pulse results in $|2\rangle_{2} \rightarrow|1\rangle_{2}$ and $|1\rangle_{2} \rightarrow-|2\rangle_{2}$ while the second pulse leads to $\left(|0\rangle_{2}+|1\rangle_{2}\right) / \sqrt{2} \rightarrow|0\rangle_{2}$ and $\left(-|0\rangle_{2}+|1\rangle_{2}\right) / \sqrt{2} \rightarrow|1\rangle_{2}$ but nothing to the state $|2\rangle_{2}$. Based on these results, one can have the following state transformations:

$$
\begin{aligned}
& \left(|0\rangle_{2}+|2\rangle_{2}\right) / \sqrt{2} \stackrel{p 1}{\rightarrow}\left(|0\rangle_{2}+|1\rangle_{2}\right) / \sqrt{2} \stackrel{p 2}{\rightarrow}|0\rangle_{2}, \\
& \left(-|0\rangle_{2}+|2\rangle_{2}\right) / \sqrt{2} \stackrel{p 1}{\rightarrow}\left(-|0\rangle_{2}+|1\rangle_{2}\right) / \sqrt{2} \stackrel{p 2}{\rightarrow}|1\rangle_{2}, \\
& |1\rangle_{2} \stackrel{p 1}{\rightarrow}-|2\rangle_{2} \stackrel{p 2}{\rightarrow}-|2\rangle_{2},
\end{aligned}
$$

where the first transformation is obtained after the first pulse while the second transformation is achieved after the second pulse.

Note that the $p 1, p 2, p 3$, and $p 4$ above represent the first, second, third, and fourth pulses, respectively.

\section{CONFICT OF INTEREST:}

The authors declare that they have no confict of interest.

[1] Shor, P.W.: in Proceedings of the 35th Annual Symposium on Foundations of Computer Science (IEEE Computer Society Press, Santa Fe, NM, 1994)

[2] Ekert, A.K.: Quantum cryptography based on Bell's theorem. Phys. Rev. Lett. 67, 661 (1991) 
[3] Bennett, C.H., Brassard, G., Crepeau, C., Jozsa, R., Peres, A., Wootters, W.K.: Teleporting an unknown quantum state via dual classical and Einstein-Podolsky-Rosen channels. Phys. Rev. Lett. 70, 1895 (1993)

[4] Schumacher, B.: Sending entanglement through noisy quantum channels. Phys. Rev. A 54, 2614 (1996)

[5] Buzek, V., Hillery, M.: Quantum copying: Beyond the no-cloning theorem. Phys. Rev. A 54, 1844 (1996)

[6] You, J.Q., Tsai, J.S., Nori, F.: Controllable manipulation of macroscopic quantum states in coupled charge qubits. Phys. Rev. B 68, 024510 (2003)

[7] Wei, L.F., Liu, Y.X., Nori, F.: Testing Bell's inequality in constantly coupled Josephson circuits by effective single-qubit operations. Phys. Rev. B 72, 104516 (2005)

[8] Wei, L.F., Liu, Y.X., Nori, F.: Generation and control of Greenberger-Horne-Zeilinger entanglement in superconducting circuits. Phys. Rev. Lett. 96, 246803 (2006)

[9] Wei, L.F., Liu, Y.X., Storcz, M.J., Nori, F.: Macroscopic Einstein-Podolsky-Rosen pairs in superconducting circuits. Phys. Rev. A 73, 052307 (2006)

[10] Yang, C.P., Chu, S.I., Han, S.: Possible realization of entanglement, logical gates, and quantum-information transfer with superconducting-quantum-interference-device qubits in cavity QED. Phys. Rev. A 67, 042311 (2003)

[11] You, J.Q., Nori, F.: Quantum information processing with superconducting qubits in a microwave field. Phys. Rev. B 68, 064509 (2003)

[12] Blais, A., Huang, R.S., Wallraff, A., Girvin, S.M., Schoelkopf, R.J.: Cavity quantum electrodynamics for superconducting electrical circuits: An architecture for quantum computation. Phys. Rev. A 69, 062320 (2004)

[13] You, J.Q., Nori, F.: Superconducting Circuits and Quantum Information. Phys. Today 58, 42 (2005)

[14] Clarke, J., Wilhelm, F.K.: Superconducting quantum bits. Nature 453, 1031 (2008)

[15] You J Q and Nori F: Atomic physics and quantum optics using superconducting circuits, Nature 474, 589 (2011)

[16] Xiang, Z.L., Ashhab, S.,You, J.Q., Nori, F.: Hybrid quantum circuits: Superconducting circuits interacting with other quantum systems. Rev. Mod. Phys. 85, 623 (2013)

[17] Gu, X., Kockum, A.F., Miranowicz, A., Liu, Y.X., Nori, F.: Microwave photonics with superconducting quantum circuits. Phys. Rep. 718-719, pp. 1-102 (2017)

[18] Blais, A., Maassen van den, B.A., Zagoskin, A.M.: Tunable Coupling of Superconducting Qubits. Phys. Rev. Lett. 90, 127901 (2003)

[19] Zhu, S.L., Wang, Z.D., Zanardi, P.: Geometric Quantum Computation and Multiqubit Entanglement with Superconducting Qubits inside a Cavity. Phys. Rev. Lett. 94, 100502 (2005)

[20] Feng, W., Wang, P., Ding, X., Xu, L., Li, X.Q.: Generating and stabilizing the Greenberger-Horne-Zeilinger state in circuit QED: Joint measurement, Zeno effect, and feedback. Phys. Rev. A 83, 042313 (2011)

[21] Aldana, S., Wang, Y. D., Bruder, C.: Greenberger-Horne-Zeilinger generation protocol for N superconducting transmon qubits capacitively coupled to a quantum bus. Phys. Rev. B 84, 134519 (2011)

[22] Yang, C.P., Su, Q.P., Zheng, S.B., Nori, F.: Entangling superconducting qubits in a multi-cavity system. New J. Phys. 18, 013025 (2016)

[23] Yang, C.P., Su, Q.P., Zheng, S.B., Han S.: Generating entanglement between microwave photons and qubits in multiple cavities coupled by a superconducting qutrit. Phys. Rev. A 87, 022320 (2013)

[24] Fedorov, A., Steffen, L., Baur, M., da Silva, M.P., Wallraff, A.: Implementation of a Toffoli gate with superconducting circuits. Nature 481, 170 (2012)

[25] Leek, P.J., Filipp, S., Maurer, P., Baur, M., Bianchetti, R., Fink, J.M., Göppl, M., Steffen, L., Wallraff, A.: Using sideband transitions for two-qubit operations in superconducting circuits. Phys. Rev. B 79, 180511(R) (2009)

[26] DiCarlo, L., Chow, J.M., Gambetta, J.M., Bishop, L.S., Johnson, B.R., Schuster, D.I., Majer, J., Blais, A., Frunzio, L., Girvin, S.M., Schoelkopf, R.J.: Demonstration of two-qubit algorithms with a superconducting quantum processor. Nature 460, $240(2009)$

[27] Ansmann, M., Wang, H., Bialczak, R.C., Hofheinz, M., Lucero, E., Neeley, M., O’Connell, A.D., Sank, D., Weides, M., Wenner, J., Cleland, A.N., Martinis, J.M.: Violation of Bell's inequality in Josephson phase qubits. Nature 461, 504 (2009).

[28] Chow, J.M., DiCarlo, L., Gambetta, J.M., Nunnenkamp, A., Bishop, L.S., Frunzio, L., Devoret, M.H., Girvin, S.M., Schoelkopf, R.J.: Detecting highly entangled states with a joint qubit readout. Phys. Rev. A 81, 062325 (2010)

[29] DiCarlo, L., Reed, M.D., Sun, L., Johnson, B.R., Chow, J.M., Gambetta, J.M., Frunzio, L., Girvin, S.M., Devoret, M.H., Schoelkopf, R.J.: Preparation and measurement of three-qubit entanglement in a superconducting circuit. Nature 467, $574(2010)$

[30] Song, C., et al.: 10-qubit entanglement and parallel logic operations with a superconducting circuit, arXiv:1703.10302

[31] Barends, R., et al.: Superconducting quantum circuits at the surface code threshold for fault tolerance. Nature 508, 500 (2014).

[32] Cirac, J.I., Zoller, P.: Preparation of macroscopic superpositions in many-atom systems. Phys. Rev. A 50, 2799(R) (1994)

[33] Gerry, C.C.: Preparation of multiatom entangled states through dispersive atom-cavity-field interactions. Phys. Rev. A 53, 2857 (1996)

[34] Zheng, S.B.: One-Step Synthesis of Multiatom Greenberger-Horne-Zeilinger States. Phys. Rev. Lett. 87, 230404 (2001)

[35] Zheng, S.B.: Quantum-information processing and multiatom-entanglement engineering with a thermal cavity. Phys. Rev. A 66, $060303(2002)$

[36] Duan, L.M., Kimble, H.: Efficient Engineering of Multiatom Entanglement through Single-Photon Detections. Phys. Rev. Lett. 90, $253601(2003)$

[37] Wang, X., Feng, M., Sanders, B.C.: Multipartite entangled states in coupled quantum dots and cavity QED. Phys. Rev. 
A 67, $022302(2003)$

[38] Yang, W., Xu, Z., Feng, M., Du, J.: Entanglement of separate nitrogen-vacancy centers coupled to a whispering-gallery mode cavity. New J. Phys. 12, 113039 (2010)

[39] Chen, Q., Yang, W., Feng, M., Du, J.: Entangling separate nitrogen-vacancy centers in a scalable fashion via coupling to microtoroidal resonators. Phys. Rev. A 83, 054305 (2011)

[40] Koch, J., et al.: Charge-insensitive qubit design derived from the Cooper pair box. Phys. Rev. A 76, 042319 (2007)

[41] Yu, Y., Han, S. private communication

[42] Clarke, J., Wilhelm, F.K.: Superconducting quantum bits. Nature 453, 1031 (2008)

[43] Neeley, M., et al.: Process tomography of quantum memory in a Josephson-phase qubit coupled to a two-level state. Nat. Phys. 4, 523 (2008); Zagoskin, A.M., Ashhab, S., Johansson, J.R., Nori, F.: Quantum two-level systems in Josephson junctions as naturally formed qubits. Phys. Rev. Lett. 97, 077001 (2006).

[44] Leek, P.J., et al.: Using sideband transitions for two-qubit operations in superconducting circuits. Phys. Rev. B 79, 180511(R) (2009)

[45] Strand, J.D., et al.: First-order sideband transitions with flux-driven asymmetric transmon qubits. Phys. Rev. B 87, 220505(R) (2013)

[46] Xiang, Z.L., Lü, X.Y., Li, T.F., You, J.Q., Nori, F.: Hybrid quantum circuit consisting of a superconducting flux qubit coupled to a spin ensemble and a transmission-line resonator. Phys. Rev. B 87, 144516 (2013)

[47] Neumann, P., Kolesov, R., Jacques, V., Beck, J., Tisler, J., Batalov, A., Rogers, L., Manson, N.B., Balasubramanian, G., Jelezko, F., Wrachtrup, J.: Excited-state spectroscopy of single NV defects in diamond using optically detected magnetic resonance. New J. Phys. 11, 013017 (2009)

[48] Pradhan, P., Anantram, M.P., Wang, K.L.: Quantum computation by optically coupled steady atoms/quantum-dots inside a quantum electro-dynamic cavity. arXiv:quant-ph/0002006

[49] Yang, C.P., Su, Q.P., Zheng, S.B., Nori, F., Han, S.: Entangling two oscillators with arbitrary asymmetric initial states. Phys. Rev. A 95, 052341 (2017)

[50] DiCarlo, L., et al.: Preparation and measurement of three-qubit entanglement in a superconducting circuit. Nature 467, $574(2010)$

[51] Baur, M., Filipp, S., Bianchetti, R., Fink, J.M., Göppl, M., Steffen, L., Leek, P.J., Blais, A., Wallraff., A.: Measurement of Autler-Townes and Mollow Transitions in a Strongly Driven Superconducting Qubit. Phys. Rev. Lett. 102, 243602 (2009)

[52] Rigetti, C., et al.: Superconducting qubit in waveguide cavity with coherence time approaching 0.1 ms, Phys. Rev. B 86, 100506(R) (2012).

[53] Chang, J.B., et al.: Improved superconducting qubit coherence using titanium nitride. Appl. Phys. Lett. 103, 012602 (2013)

[54] Chow, J.M., et al.: Implementing a strand of a scalable fault-tolerant quantum computing fabric. Nat. Commun. 5, 4015 (2014)

[55] Peterer M.J., et al.: Coherence and decay of higher energy levels of a superconducting transmon qubit. Phys. Rev. Lett. 114, 010501 (2015).

[56] You, J. Q., et al.: Low-decoherence flux qubit. Phys. Rev. B 75, 140515(R) (2007)

[57] Sank D., et al.: Measurement-Induced State Transitions in a Superconducting Qubit: Beyond the Rotating Wave Approximation. Phys. Rev. Lett. 117, 190503 (2016).

[58] Chen, W., Bennett, D.A., Patel, V., Lukens, J.E.: Substrate and process dependent losses in superconducting thin film resonators. Supercond. Sci. Technol. 21, 075013 (2008)

[59] Leek, P.J., Baur, M., Fink, J.M., Bianchetti, R., Steffen, L., Filipp, S., Wallraff, A.: Cavity quantum electrodynamics with separate photon storage and qubit readout modes. Phys. Rev. Lett. 104, 100504 (2010)

[60] Yang, C.P., Chu, S.I., Han, S.: Quantum Information Transfer and Entanglement with SQUID Qubits in Cavity QED: A Dark-State Scheme with Tolerance for Nonuniform Device Parameter. Phys. Rev. Lett. 92, 117902 (2004)

[61] Yang, C.P., Han, S.: n-qubit-controlled phase gate with superconducting quantum-interference devices coupled to a resonator, Phys. Rev. A 72, 032311 (2005) 